\title{
NASA LANGLEY AND NLR RESEARCH OF DISTRIBUTED AIR/GROUND TRAFFIC MANAGEMENT
}

\author{
Mark G. Ballin * \\ NASA Langley Research Center, Hampton, Virginia \\ Jacco M. Hoekstra \\ National Aerospace Laboratory - NLR, Amsterdam, The Netherlands \\ David J. Wing \\ Gary W. Lohr \\ NASA Langley Research Center, Hampton, Virginia
}

\begin{abstract}
$\underline{\text { Abstract }}$
Distributed Air/Ground Traffic Management (DAG$\mathrm{TM}$ ) is a concept of future air traffic operations that proposes to distribute information, decision-making authority, and responsibility among flight crews, the air traffic service provider, and aeronautical operational control organizations. This paper provides an overview and status of DAG-TM research at NASA Langley Research Center and the National Aerospace Laboratory of The Netherlands. Specific objectives of the research are to evaluate the technical and operational feasibility of the autonomous airborne component of DAG-TM, which is founded on the operational paradigm of free flight. The paper includes an overview of research approaches, the airborne technologies under development, and a summary of experimental investigations and findings to date. Although research is not yet complete, these findings indicate that free flight is feasible and will significantly enhance system capacity and safety. While free flight cannot alone resolve the complex issues faced by those modernizing the global airspace, it should be considered an essential part of a comprehensive air traffic management modernization activity.
\end{abstract}

\section{$\underline{\text { Introduction }}$}

The aviation user community has identified a need for significantly increasing airspace capacity and the flexibility of aircraft operations. This need and the introduction of new surveillance concepts have led to a new operational paradigm, "free flight," in which reliance on centralized air traffic management is reduced in favor of distributed management. In 1995, RTCA Task Foree 3 defined free flight as a safe and efficient flight operating capability under instrument flight rules in which operators have the freedom to select their path and speed in real time. ${ }^{1}$ In 1997, the NASA Advanced Air Transportation Technologies

* Associate Fellow, AIAA
Project (AATT) began developing and exploring the concept of Distributed Air/Ground Traffic Management (DAG-TM). NASA experience in developing airborne and ground-based decision support systems was used to provide a detailed definition of the broad vision of free flight. DAG-TM is based on the premise that large improvements in system capacity as well as flexibility and efficiency for the airspace user will be enabled through

- sharing information related to flight intent, traffic, and the airspace environment,

- collaborative decision making among all involved system participants, and

- distributing decision authority to the most appropriate decision maker. ${ }^{2}$

Flight crews, the air traffic service provider (ATSP), and aeronautical operational control organizations interact as both information suppliers and users, thereby enabling collaboration and cooperation in all levels of traffic management decision making. Distributing decision-making authority may be the key enabler in multiplying the capacity of National Airspace System by minimizing the occurrence of human workload bottlenecks. It offers the potential of a linearly scalable system that accommodates an increase in demand through a proportional increase in infrastructure and human decision-making capability, whereby each additional aircraft contributes actively to the traffic management solution. System-wide reliability and safety improvements may also result from the increased redundancy of traffic management capability.

Under the sponsorship of AATT, NASA Langley Research Center (Langley), NASA Ames Research Center (Ames), and the National Aerospace Laboratory of the Netherlands (NLR) have collaborated in research and development to explore the feasibility of DAGTM. Two of the fifteen DAG-TM concept elements

$-1-$

American Institute of Aeronautics and Astronautics 
focus on the original vision of free flight as a distributed airborne traffic management concept where the flight crew has independent authority to perform tasks currently associated with an air traffic controller. DAG-TM Concept Element 5 envisions aircraft operating autonomously in constrained en route and unconstrained terminal-arrival environments, and Concept Element 11 envisions flight crews contributing actively in maximizing airport arrival throughput when instrument approaches are in use. Both concept elements are based on the premise of Reference 1 that flight crews should have capability and authority to self-separate from other aircraft by adjusting their self-selected trajectories, thereby freeing air traffic service providers to concentrate on traffic flow management.

This paper provides an overview and status of ongoing research at Langley and the NLR in the areas described by these two concept elements. Specific objectives of the research are to evaluate the technical and operational feasibility of the autonomous airborne component of DAG-TM and to develop and validate the enabling airborne technologies and procedures that will be required. The paper includes an overview of the research approaches, the airborne technologies under development, and a summary of experimental investigations and findings to date. In the paper, the term "free flight" is used to refer to the capability of aircraft to operate autonomously as a component of a larger DAG-TM concept rather than as the complete concept in itself.

\section{$\underline{\text { Research and Development Approach }}$}

Published position statements and pre-existing research provide an ambiguous assessment of free flight feasibility. The Task Force 3 report states that, in addition to autonomous airborne capability for separation, ground-based automation and restrictions will be used to resolve tactical conflicts, to manage traffic flows in congested airspace, to prevent unauthorized entry into special use airspace, and to assure safety of flight, ${ }^{1}$ which suggests that feasibility and safety depend on ground capabilities. The task force stipulated that free flight must be at least as safe as today's operations and implied that ground involvement and restrictions are adequate to provide this. To alleviate concerns, the National Academy of Science's National Research Council coordinated an activity to gather expert opinion regarding safety implications of the task force's vision. The resulting opinion stated that the safe distribution of traffic management responsibility is very difficult, if not impossible. ${ }^{3,4}$ However, other research indicates that free flight is not difficult and that it may in fact be safer than ground-based separation assurance. ${ }^{5,6}$ These contradictions may have been the result of compartmentalized perspectives and expertise, leading to uncoordinated assessments and inconsistent use of enabling technology.

To resolve these ambiguities, a balanced research approach was chosen that focuses primarily on determining the limits of feasibility and the technology requirements of the concept elements. Under this approach, the following steps are taken concurrently and iteratively: a set of hypotheses is developed, the future airspace system is modeled in simulation, technology prototypes and operational procedures are developed, and evaluation is performed. The hypothesis set includes nominal operations, rarenominal operations (events or conditions that stress the concept or define the limits of feasibility), and failuremode operations. If feasibility is established for airborne operations, further activities will focus on the issues involved in integrating the airborne components with ATSP components. Benefits and safety aspects, while important, have only been characterized at a high level until feasibility is established. High-level benefits assessments are in progress, but are not discussed in this paper.

Because decision support automation technology is an integral part of the DAG-TM concept, it is necessary to develop prototype technology and procedures to make a valid determination of feasibility. Airborne decision support technology is therefore under development. ${ }^{7,8}$ Free flight operations may require flight deck systems that support the flight crew in making trajectory management decisions. Requirements may include the capability to collect, fuse, and present relevant information; analyze surveillance and constraint data for potential airspace or traffic conflicts; calculate conflict resolution options that optimize specified parameters, such as fuel burn; provide tactical information for conflict-free maneuvering; and analyze over-constrained problems for viable solutions. These and other capabilities that may be critical for free flight in complex traffic and airspace environments do not exist in current flight deck systems.

While the airborne decision-aiding systems described above may improve crew planning capabilities and reduce workload, they may also increase the cost of equipping aircraft for free flight operations and be difficult to retrofit. In addition, they may place an increased burden on the future communication, navigation, and surveillance (CNS) infrastructure that will enable aircraft to exchange needed information. Both of these factors may delay the transition to future free-flight operations. Therefore, it was deemed appropriate to also determine the minimum airborne requirements necessary for free flight. 
Langley and the NLR have chosen to collaborate in a complementary approach that investigates this range of capabilities. Langley is primarily investigating issues that concern advanced airborne technology, as may be required or preferred in a mature system that integrates autonomous aircraft and managed aircraft in the same airspace, integrates with ground-based systems, and performs long-term planning that may aid traffic flow. The NLR is primarily investigating technology and procedures that require minimum changes to flight deck systems and the CNS infrastructure. The NLR results are presented in the section that follows, and the NASA results are presented in the remaining two sections.

\section{$\underline{\text { Tactical Free Flight Operations }}$}

One research and development alternative attempts to determine what is minimally required to enable airborne separation assurance. Led by the NLR, the approach considers a tactical concept that resolves a predicted loss of separation with traffic (referred to as a "conflict"), but does not include a recovery of the aircraft's flight plan as part of the predetermined resolution maneuver. Since the concept assumes that the separation assurance function can be accomplished independently from strategic functions such as conformance to flow management constraints, this assumption was investigated as part of the research.

\section{Operational Concept}

As a first step, an operational concept for the experiments was defined. The advanced CNS capability assumed available is Automatic Dependent Surveillance-Broadcast (ADS-B) or a comparable capability that provides, at a minimum, basic aircraft state data at intervals on the order of one second. The operational concept has the following characteristics:

- State-based conflict detection

Only state data, i.e., position (latitude, longitude and altitude) and velocity (ground speed, track and vertical speed), are exchanged between aircraft. The conflict detection function uses no flight plan information. This was used as a null hypothesis to determine whether flight plan information would be fundamentally required for concept feasibility. A look-ahead time of five minutes was provided by the detection function.

- Cooperative conflict resolution

Rules, implemented in the conflict resolution function that advises the pilot, use the geometry of the detected conflict to determine the direction in which to maneuver. The system does not assume the intruder aircraft will maneuver, but if the intruder utilizes the same rules, it will maneuver to further increase the separation distance. Using rules rather than coordination messages is called implicit coordination, and is important in reducing requirements for data exchange between aircraft.

- $100 \%$ autonomous during cruise flight

All aircraft were assumed to have free flight capability, and no active role for the ground was included in the operational concept. This corresponds to the European concept of segregating free flight operations from managed operations into 'free flight airspace' and 'managed airspace.'

\section{Conflict Resolution Method}

Off-line traffic simulations comprising up to 400 aircraft were used to validate several methods for conflict detection and resolution. These simulated traffic densities are equivalent to ten times today's average western European density. The resolution method that proved to be most effective was based on the method described in Reference 9.

The conflict resolution algorithm uses the geometry of the closest point of approach to prevent counteracting maneuvers. Assuming both aircraft use the same system, both aircraft maneuver cooperatively, which provides implicit coordination. The calculated positions at the closest point 'repel' each other, similar to the way charged particles repel each other. As shown in Figure 1, the 'repelling force' is converted to a displacement of this predicted position in such a way that the minimum distance will be equal to the required separation. This avoidance vector is converted into advised heading and speed changes. The same principle is used in the vertical plane, resulting in an advised vertical speed and target altitude.

When both aircraft are able to maneuver cooperatively, a redundant element is introduced. If both aircraft do

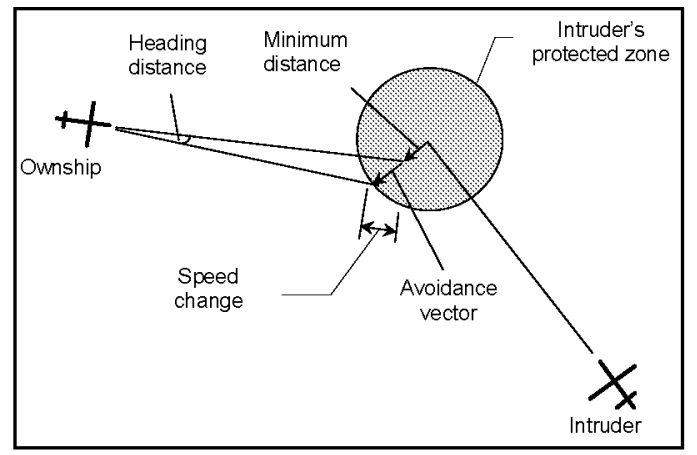

Figure 1. Graphic representation of conflict resolution method.

$-3-$

American Institute of Aeronautics and Astronautics 
maneuver, the conflict alert will disappear when each aircraft has executed part of the maneuver, indicating that no further avoiding action is required. The resulting maneuvers are typically a few degrees of heading change or about $200 \mathrm{ft} / \mathrm{min}$ of vertical speed change. Passenger comfort is not affected by these shallow maneuvers.

Complex geometries and restrictions were used to test the robustness of the method. These geometries include circular radial conflicts and several types of opposing walls of aircraft. Though they do not represent actual traffic conflict scenarios, these scenarios were designed to determine the power and limits of the conflict resolution method by providing more complex traffic constraints than would ever occur in operation.

It was found that even though the aircraft follow independent resolution advisories, a global solution to the problem arises. In the 'wall' scenarios, for example, some aircraft in the wall decelerate slightly while others accelerate, thereby opening a space for an opposing aircraft to fly through. Distributing the cooperative resolution algorithm among all involved aircraft results in an efficient and global solution, even for situations where no solution existed prior to resolution. The algorithm proved capable of resolving conflict situations for which a priority system, which forces one aircraft of a pair to perform the entire maneuver, would not have obtained a solution.

\section{Cruise Human-in-the-loop trials}

The conflict resolution algorithm used in the traffic simulation has been developed further into an airborne separation assurance system (ASAS) for a research flight simulator at the NLR. The ASAS includes a human-machine interface that has been tested in several flight simulator trials. Airline pilots have been exposed to scenarios replicating current densities ('single') up to three times the Western European density ('triple'). Only a few hours of training was found necessary.

No significant increase in workload was found during the cruise phase. The acceptability was surprisingly high and, further, the subjective safety was equal or better than today's situation. ${ }^{10}$ Figure 2 shows workload assessments for three levels of traffic density. The Rating Scale for Mental Effort (RSME) system of Reference 11 was used as a measure. The total range of the RSME measure is 0 to 130 . The single density session shows a workload rating very close to a rating of 27 found for a comparable ATC situation. These results were obtained using no flight plan information, explicit coordination procedures, priority rules, or ground-based systems.



Figure 2. Workload assessments of a simulation of en route cruise tactical free flight.

\section{Conflict Prevention System}

A conflict prevention system called "Predictive ASAS" was also developed. The system prevents short-term conflicts as a result of turns and vertical maneuvers. For safety, a conflict prevention rule was added that forbids flight crews from maneuvering to create a conflict. This rule and the Predictive ASAS display were hypothesized to be an alternative to the exchange of flight plan information as a method to prevent conflicts.

Because of the simplicity of the architecture and the resolution method, the conflict prevention system was transparent to the crew, allowing a display design as shown in Figure 3. The display shows both a horizontal and vertical resolution advisory to the pilot. Either advisory will completely resolve the conflict. The conflict prevention system draws amber and red no-go zones ('conflict prevention bands') on the heading, vertical speed, and speed scales of the displays, even if there are no conflicts for the current heading. This system was tested in a second human-in-the-loop experiment. It was found that pilots used the dynamically changing conflict prevention bands as precursors to a conflict alert and were able to exploit this to lower the number of conflict alerts. This indicates that a conflict prevention system can contribute to the traffic situation awareness of the crew.

\section{Cruise findings}

None of the studies refuted the feasibility of airborne separation during cruise, even under extremely dense and constrained traffic situations. The findings indicate that decentralizing today's ATM system can significantly increase the capacity in the en-route domain, even with minimal flight deck technology. 




Figure 3. Co-planar traffic display as used in the study. The symbology indicates a conflict (amber) and the resolution advisory (green).

Contrary to what was believed when the project started, there appears to be no fundamental requirement for exchanging intent (flight plan or mode control panel) information, although possible benefits of doing so are still acknowledged.

It was also found that the conflict resolution maneuvers had a negligible impact on flight efficiency or the ability of the aircraft to meet a time constraint. When the crews were trained to nominally use a vertical resolution maneuver instead of a horizontal one, there was no noticeable impact. This finding is important in establishing a transition procedure from free flight airspace to managed airspace. Because the planning problem and the separation problem appear to be independent, there is no fundamental difference between this transition and today's hand-over from one ground-based controller to another. This alleviates the need for complex hand-over procedures that use huge transition zones around entry points.

\section{Other flight phases}

As a next step, the basic ASAS was tested in a piloted simulation experiment for different flight phases that are more constrained than the cruise phase. Each aircraft started in cruise and proceeded through descent, arrival, and approach phases of flight. In half the runs, the terminal area was managed airspace with a basic hand-over as the transition procedure, and the crew was provided with a cockpit display of traffic information (CDTI) to monitor traffic. In the other half,

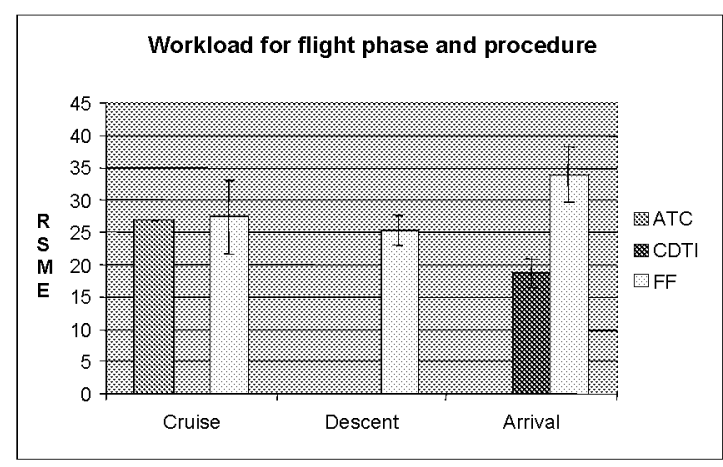

Figure 4. Workload ratings for three flight phases and three levels of airborne capability.

the flight crew was responsible for the separation until established on the localizer, and the controller established only the arrival sequence.

At the end of each run, pilots were asked to rate the mental workload of the descent and the arrival segments. These ratings and the earlier results for cruise are compared in Figure 4. Three categories of operational procedures were compared: free flight (FF); managed with a CDTI (CDTI); and managed without a CDTI (ATC).

It has often been suggested that a higher workload during the descent might inhibit free flight during that phase of operations. As can be seen in the figure, the FF descent rating is not significantly different from the FF cruise rating, at least for the initial traffic scenarios studied. During the FF arrival, the workload was found to be significantly higher than for the other phases of flight, but not high on the absolute scale, which extends to a value of 130 . Although the limits of its feasibility are not fully established, evidence suggests there is a role for airborne separation assurance in the terminal area.

In general, the arrival phase of flight is characterized by much higher workload than the cruise phase. ${ }^{12}$ In Figure 4, the workload rating for the CDTI arrival shows a dramatic impact of providing traffic information to the crew. The arrival rating is even lower than the ATC cruise scenario without CDTI. However, during merging maneuvers required in the high traffic density terminal area, the crews were occasionally not able to maintain separation. Some pilots commented that more training would be sufficient, but this is not supported by the objective data, which show no training effect of separation effectiveness during merging. Therefore, the basic ASAS retrofit will not be sufficient for complex highdensity terminal airspace. Either more sophisticated tools and procedures are required, or the terminal area

$-5-$

American Institute of Aeronautics and Astronautics 
should remain managed airspace with the addition of the CDTI to reduce crew workload. As described later, approach spacing with limited delegation may also offer solutions for capacity-constrained terminal environments.

\section{$\underline{\text { Strategic Free Flight Operations and Air/Ground }}$ $\underline{\text { Integration }}$}

\section{En-Route Strategic Airborne Operations}

Complementary to the tactical airborne operations in free flight investigated by the NLR are strategic airborne operations investigated by Langley. The main purpose for a strategic mode in free flight is to allow the flight crew to be proactively engaged in the dynamic airspace operational environment. This may require an increase of the planning horizon beyond what tactical mode enables and a capability to replan trajectories to gain operational advantage under a complex set of constraints. For cost effectiveness, strategic free flight capabilities would make maximum use of automation systems already present on most modern aircraft, such as the flight management system (FMS) and the FMS-coupled autoflight system. Just as these systems assist the crew in managing the strategic intent of the flight, i.e., specifying and enacting higherlevel goals than simply aircraft state changes, strategic free flight capabilities focus on the use of intent-based trajectories and longer look-ahead time horizons for strategic flight replanning, conflict detection and resolution, and constraint management.

In addition to that of the tactical mode, a strategic free flight capability provides functionality in the areas of information management (gathering and processing), conflict detection and alerting, and conflict resolution. Each area will be briefly described.

The objective for information management in strategic free flight is

- to gather and integrate sufficient predictive information on the operating environment to enable increased trajectory prediction accuracy and replanning time before action is required

- to make available more options for solving a given problem, and

- to allow replanned trajectories to be valid (i.e., constraints to be met) over a longer-term portion of the flight.

Information needed to support these goals includes traffic surveillance with trajectory intent, airspace system status, weather products, and assigned operational constraints to facilitate traffic flow management (TFM).
For conflict detection and alerting, the approach in strategic free flight is to use the best information available for determining whether a loss of separation with another aircraft or an encroachment on hazardous airspace is threatened and whether the flight crew should or should not take action in each particular situation. Trajectory information on traffic aircraft may include only state data for some aircraft (for instance if that aircraft is currently being maneuvered manually), or it may also include varying degrees of intent ranging from target states to FMS-level intent if the autoflight system is engaged. The determination of which trajectory information is best suited for detecting conflicts relates to several conditions, such as traffic aircraft conformance to its broadcast intent and proximity to the ownship. Performing conflict detection using both state and intent information has the potential for reducing both false and missed alerts while increasing detection accuracy over a larger lookahead time horizon. These effects, in turn, can reduce unnecessary and excessive maneuvering, thereby freeing the crew to better manage their other tasks.

With the increased look-ahead horizon for conflict detection, strategic free flight allows problems to be solved with strategic solutions. A strategic solution is a set of complete resolution maneuvers including return to course that can be calculated and evaluated by the crew before the first maneuver is initiated. This complete solution can also be integrated into the FMS flight plan, and the maneuver can be accomplished while remaining in FMS guidance mode during the entire event. An important community benefit of doing so is that the modified FMS flight plan is broadcast as new ownship intent, thereby increasing the predictability of the ownship to other airborne and ground-based observers. An aircraft that resolves conflicts tactically by exiting FMS guidance mode would not be as predictable, since the intent of that aircraft is not shared. An additional benefit to strategic resolutions is the ability to simultaneously incorporate all operational constraints into the solution, including those unrelated to the specific conflict. Examples of such constraints are required time of arrival (RTA) flow constraints issued by the ATSP, ownship performance limitations, and company-specific flight optimization strategies. Derived user benefits from incorporating these operational constraints into resolution maneuvers may include improved schedule conformance and reduced fuel burn.

A hypothetical example of strategic free flight problem solving is shown in Figure 5. The trajectories of two aircraft are in conflict in en-route airspace. Assuming a priority system is in effect and the aircraft to the right (Aircraft ' $B$ ') has priority, aircraft ' $A$ ' must replan its 


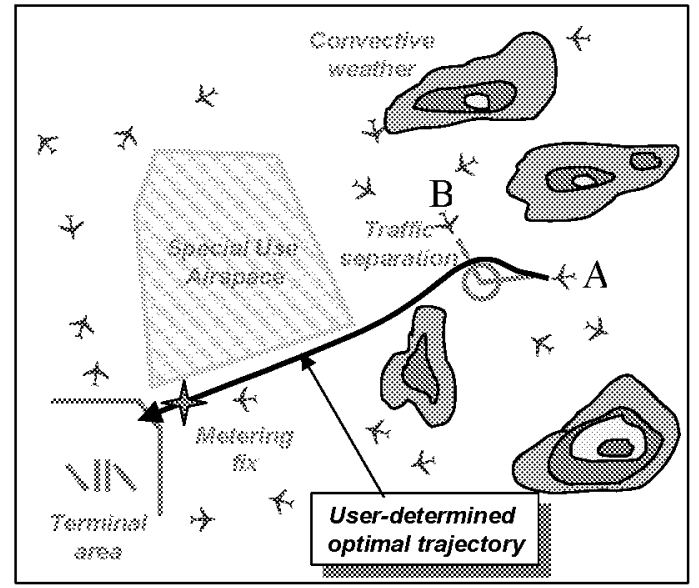

Figure 5. Hypothetical example scenario for strategic planning.

trajectory such that separation does not decrease below the accepted regulatory standard for safety (accounting for applicable navigation and surveillance uncertainties). However, several other constraints on the aircraft ' $\mathrm{A}$ ' trajectory are also present. The aircraft is approaching the terminal environment, and the ATSP has issued a TFM constraint to aircraft ' $\mathrm{A}$ ' in the form of an RTA crossing restriction at the arrivalmetering fix. An SUA is active to the north of the fix, and convective weather cells are forecast for the vicinity. Furthermore, additional crossing traffic below and to the left may impact the decision on whether an early descent is advisable.

Assuming these constraints are input to the flight crew's decision-aiding automation, the FMS flight plan can be modified by computing an RNAV path between present position and the destination that adheres to each of these constraints. This computation can be initiated as soon as the conflict is identified and provided to the flight crew, either automatically or on request. In the example, a resolution trajectory is calculated that diverts the aircraft to the right and behind the conflicting aircraft, and then the return path is calculated to miss the SUA and weather cell while minimizing excess air miles flown. Airspeed and top of descent are adjusted as necessary to meet the crossing restriction. A diversion to the south is not offered because of the size of the weather cell. A climb is not recommended because it would not be consistent with the impending arrival, and an early descent would create a new traffic conflict. The flight crew is able to review the strategic plan and modify it if necessary. If it is deemed acceptable, the crew activates the route in the FMS, and the route is broadcast as new intent.

Developing and Evaluating Strategic Free Flight Researchers at Langley are developing and evaluating the capability for strategic free flight. The development activity involves defining and creating a software prototype of a flight crew decision support tool that facilitates the strategic operations described in the previous section. This tool, referred to as the Autonomous Operations Planner (AOP), provides for management of operational constraints and user preferences. It processes surveillance and airspace status information, and searches for conflicts among several relevant trajectory combinations between ownship and traffic aircraft. These combinations include state and several forms of intent, as well as conflicts with airspace hazards. Crew alerting is provided based on threat priority and required crew action. Strategic and tactical conflict resolution advisories are provided to the crew, with options from which to chose. The strategic resolutions are iterated through the FMS to ensure the trajectory recommendations are within aircraft performance limits and that operational constraints such as an RTA can be met. See reference 7 for a more complete description of $\mathrm{AOP}$ capabilities.

An example of a highly constrained conflict scenario with a strategic AOP resolution is shown in Figure 6. In the scenario, the ownship flight plan passes between two SUA regions that constrain the available solution space. The broadeast intent from a traffic aircraft is in conflict with the ownship flight plan, and the conflicting portion of the flight plan is shown to the pilot. In this example, AOP only considered lateral path-stretch solutions to the conflict. An AOPgenerated conflict resolution trajectory involving a path-stretch maneuver to the right is shown for pilot review. This trajectory has been determined to be free of any traffic conflicts, to adhere to the airspace constraints, and to be flyable by the aircraft. Note that the outboard waypoint is a fly-by waypoint, and that given the aircraft's turn radius, the aircraft itself will not pass into the SUA. Future builds of the AOP software will also consider the vertical and speed degrees of freedom.

Some aspects of strategic free flight were recently evaluated in a human-in-the-loop simulation conducted in the Air Traffic Operations Laboratory at Langley. ${ }^{13,14}$ Operations were conducted under conditions of low and high operational complexity (traffic and airspace hazard density) with operational constraints including RTA adherence and airspace hazard avoidance.

Strategic free flight was found to be effective in reducing unnecessary maneuvering during conflict situations where the intruder's intended maneuvers would resolve the conflict. Scenario case studies illustrated the need for flight restrictions to prevent the 


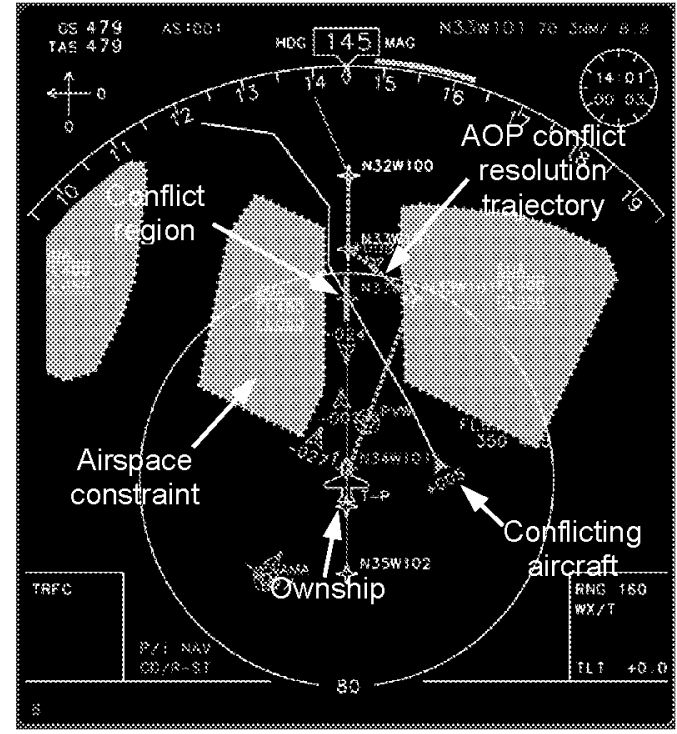

Figure 6. Example conflict scenario with a strategic $A O P$ conflict resolution (on an MD-11 novigation display).

creation of new conflicts through maneuvering, as discussed in the previous section. The case studies have also shown the need for an improved user interface design that appropriately focuses the pilot's attention on conflict prevention information. Pilot realtime assessment of maximum workload indicated minimal sensitivity to operational complexity, providing further evidence that pilot workload is not the limiting factor for feasibility of an en-route distributed traffic management system, even under highly constrained conditions.

\section{Strategic Air-Ground Integration}

The discussion thus far has focused primarily on the characteristics and advantages of strategic free flight from an airborne perspective. When considering integration of the airborne and ground components in air traffic operations, the strategic mode for free flight provides some additional key benefits. These benefits would be particularly important where autonomous and managed aircraft are intermixed rather than segregated into 'free flight airspace' and 'managed airspace'.

As stated earlier, the strategic free flight broadcast of intent information provides increased predictability of the operational environment to airborne recipients of that information. This predictability is also of great importance to the ATSP in its role of providing for orderly flow through the airspace and into and out of high-demand terminal environments. Metering would be a principal technique for controlling the flow; however the ability to meter may be highly dependent on the ability to dynamically predict the demand throughout the airspace. As more aircraft operate in the autonomous mode and exercise the freedom to modify their trajectories as needed, the need for these aircraft to contribute to system predictability by operating strategically increases, which includes broadcasting intent and making fewer but more strategic intent changes.

Strategic free flight also benefits air-ground integration in conflict situations and managed-aircraft trajectory control. For non-segregated operations to be feasible, it is likely that free flight operations (for which the controller is not responsible) must have the appearance to the controllers of occurring largely on a noninterference basis. This has several implications. First, conflicts between autonomous aircraft should generally be resolved before the controller (or monitoring automation) sees the conflict. Second, changes in intent or maneuvers by autonomous aircraft must not create new conflicts with managed aircraft. For both conditions to be met requires autonomous aircraft to use a longer look-ahead horizon than the controller team, which often plans ahead many minutes. The second consideration is particularly important in that the noninterference operations provide some protection to the controller from the maneuvering activities of autonomous aircraft. This protection is likely a key feasibility requirement because it allows the controller to effectively and strategically plan the managed aircraft trajectories.

An important aspect of air-ground integration is handling situations for which an autonomous aircraft can no longer operate autonomously and must transition to managed status. Assuming that the need for the transition may occur unexpectedly and possibly at an inopportune moment for the receiving controller, the operational concept must allow for a finite and noninstantaneous transition time before the controller can assume responsibility. An analogy would be a VFR aircraft requesting a pop-up IFR clearance; until the clearance can be issued, the pilot must continue to operate under VFR and provide for its own separation. Strategic free flight can facilitate this transition in that the autonomous aircraft, before the failure, would be established on a trajectory that was determined to be conflict free potentially for tens of minutes into the future. The increased look-ahead and traffic-aircraft intent information of strategic free flight would provide a significant amount of protected time to accomplish the transition.

\section{$\underline{\text { Feasibility }}$}

The research and development performed thus far support the feasibility of strategic autonomous aircraft operations. It has been learned that autonomous aircraft operations scale well with traffic density. En route

$-8-$

American Institute of Aeronautics and Astronautics 
workload in nominal situations is not a feasibility issue, even in very high traffic densities. Given appropriate decision-support automation, pilots can strategically resolve conflicts in complex environments that include TFM, airspace, and performance constraints.

Combined use of state and intent provides valuable operational benefits, although intent may not be a fundamental requirement for feasibility. Evidence indicates that autonomous operations can be reliably performed without the need for controller intervention.

While additional challenges to feasibility remain and have yet to be fully explored, no insurmountable impediments have yet been discovered for free flight aircraft operations in all environments except highlyconstrained terminal areas. More research is needed on air-ground integration to determine the feasibility of integrating mixed-equipage operations in the same airspace, to study failure-mode transitions between equipage status levels, and to identify the sensitivity of controller workload to large increases in traffic density.

\section{Capacity-Constrained Terminal Area Operations}

The inherent dynamic and highly constrained nature of terminal area operations requires an approach different from that employed for the en route domain. Operations in the busy terminal area are characterized by:

- constraints on the volume and configuration of airspace caused by number and geometry of runways, surface topology, and environmental considerations such as noise

- dynamically changing operations caused by changes in wind direction, meteorological conditions, availability of individual runways, and the relative demand for arrival, departure, and surface operations

- varying aircraft performance and equipage levels

The high-density airports in the current system require solutions that will ultimately increase capacity. If maximization of airport throughput dominates other operational needs, a distributed air/ground traffic management approach offers several advantages. Increasing airborne responsibility for trajectory management can provide increased conformance with constraints that maximize throughput. Flight crews are capable of high precision in managing their own trajectory. Increased precision leads to a reduction in spacing buffers and hence higher throughput. A ground-based controller cannot achieve such precision, and he must simultaneously manage numerous aircraft.
Increased positioning capability obtained by providing crews with limited maneuvering authority also results in fewer missed arrival slots over time. A further advantage is the growth potential provided for today's underutilized airports that will see increased demand in the future. Because each aircraft brings with it a significant portion of the needed CNS infrastructure and human-decision-making capability, minimal ground infrastructure additions will be required for these terminal areas as demand increases.

As for en route operations, the advanced CNS capability assumed for the terminal area research is ADS-B or its equivalent. Additional ADS-B information content such as planned final approach speeds, wind data, and intent information may be required depending on the mode of operation.

\section{Concept Overview}

Distributed air/ground terminal area operations are being investigated through the definition of three operational modes: 'in-trail spacing,' 'merging operations,' and 'maneuvering.' For all modes, procedures involve limited delegation of specific responsibilities by the ATSP to each participating aircraft. Each of these modes embodies characteristics that allow for implementation independent of the others or in combination, depending on the specific operational needs of the environment. Figure 7 depicts a general overview of the three operational modes, which are defined as follows:

- In-Trail Spacing

The in-trail spacing mode is based on the ATSP providing a spacing interval to the following aircraft and issuing a clearance for the flight crew to adhere to algorithm-generated speed cues to achieve the spacing. Spacing is time-based to account for differing final approach speeds and wind environments. The aircraft follows the same flight path as the aircraft immediately preceding it, following either a defined route or a flight path history (i.e., following the ground track of the lead aircraft, which is provided on an onboard map display).. In-trail spacing can be applied at any point in the terminal area, and should be applied as far in advance as possible to maximize throughput benefits. In-trail spacing may also have applications in the en route and oceanic domains.

- Merging

Aircraft arriving on different routes that merge at a common point are appropriately spaced at that point, based on an RTA that is either assigned or computed based on the lead aircraft's estimated 


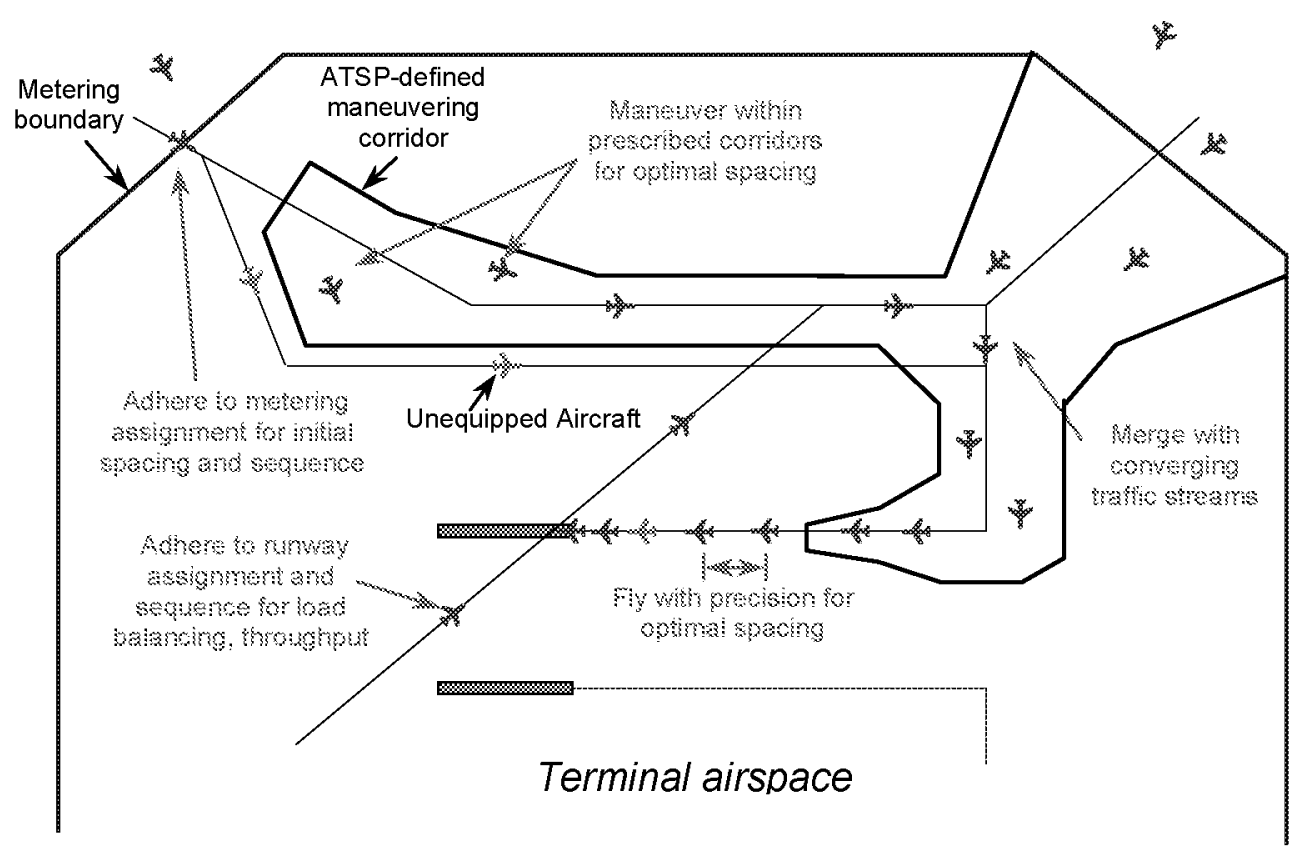

Figure 7. DAG-TM terminal arrival concept element.

time of arrival at the point. This mode assumes a pre-defined route environment where a sufficient number of routes exist to allow aircraft to be scheduled ahead or behind traffic on separate routes and arrive properly spaced at the merge point. Under a less rigid application, aircraft could pass common boundaries, as opposed to points, with appropriate time separations.

\section{- Maneuvering}

To enable a greater spacing dynamic range than is possible with speed adjustments alone, aircraft are given the flexibility to define their own routes within prescribed airspace, provided that systemimposed constraints are met. Although the term 'maneuvering' might suggest last minute changes in heading or speed, it is envisioned that advanced planning (prior to terminal area entry) would result in a near-optimized, stable flight path through the terminal area. Assuming an adequate conflict detection and resolution capability is available, more dynamic maneuvers could potentially also be executed. Future research will determine if this is feasible and provides benefits.

\section{In-Trail Spacing Operations}

Langley research of time-based in-trail spacing dates back to the 1970s. The results of several simulator studies indicated that the potential existed for capacity increases ${ }^{15,16}$ although some issues related to displays and supporting data links remained to be addressed in the development of an acceptable operation.
The investigations began with a complete definition the concept and procedures, including a checklist for the flight crew and phraseologies specific to the new approach spacing operation. The concept includes use of an airborne decision support tool, which is made up of two components:

- A specialized algorithm, referred to as Advanced Terminal Area Approach Spacing (ATAAS), generates speed guidance to achieve a desired spacing interval at the runway threshold. Described in Reference 17, ATAAS has been refined through extensive Monte Carlo analysis.

- Supporting displays provide a crew interface.

A nominal speed profile is provided that reflects speeds typically used in arrival operations, and is included as part of a charted arrival procedure. This procedure can be used by all arriving aircraft to follow the nominal speed schedule, regardless of their ability to perform an approach spacing operation. Since the ultimate goal is to provide maximum achievable system throughput in a stable and acceptable manner, the ATAAS speed guidance is limited to $\pm 10 \%$ of the nominal profile so that system stability and pilot acceptability are maintained.

Figure 8 provides an example of some of the display symbology. The Navigation Display provided information on the ATAAS guidance and aircraft spacing status. The information included a data block containing currently entered ATAAS data and lead 


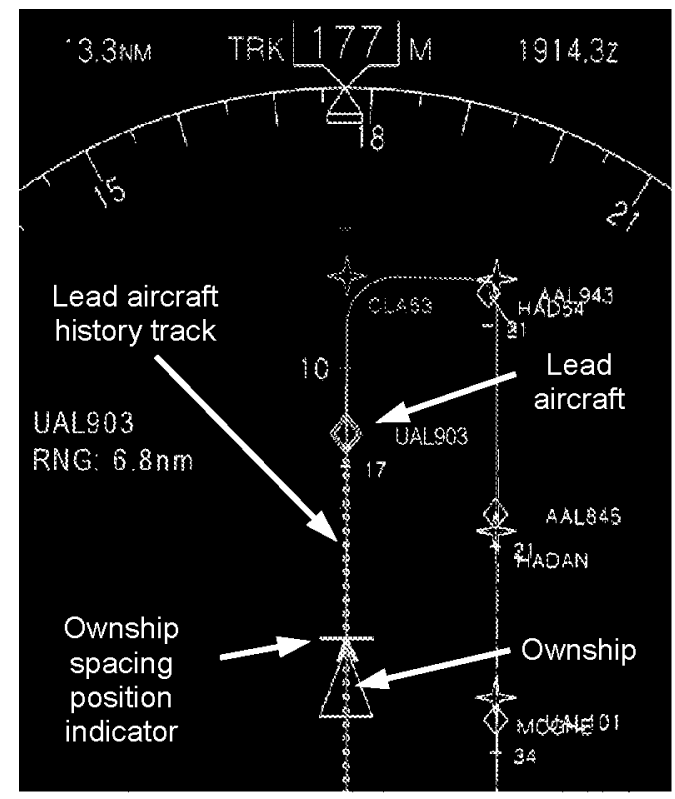

Figure 8. B757 navigation display with approach spacing symbology.

aircraft range, a spacing position indicator; and lead aircraft history dots, showing the ground track taken by that aircraft.

A piloted simulation study was conducted in January 2002 to validate the results of the Monte Carlo analysis and to evaluate pilot workload and acceptability. The study was conducted in a full mission B757 simulator with B757-rated airline pilots. The pilots were issued a clearance to follow the ATAAS speeds, allowing them to fly the pre-defined path through the terminal area while adhering to speed guidance provided by the ATAAS algorithm. The arrivals were flown using one of three speed management modes: ATAAS-coupled autothrottles, pilot control of speed through the Mode Control Panel, and manual throttle control

Data collected for the study included aircraft state data, subjective (questionnaire) data, workload ratings using the NASA-TLX (Task Load Index) method, ${ }^{18}$ and eyetracker data. The results from all three sources of data were very positive. As shown in Figure 9, aircraft delivery performance at the runway threshold was \pm 1 second for autothrottle-coupled runs, and within 5 seconds for the other two speed management modes. These times correspond to a threshold crossing accuracy on the order of \pm 200 feet for the autothrottlecoupled mode and within 1100 feet for manual modes.

The subjective data also yielded positive results, with high positive ratings from the pilots regarding overall acceptability, amount of heads-down time, and confidence in the guidance. Eye tracker data indicated

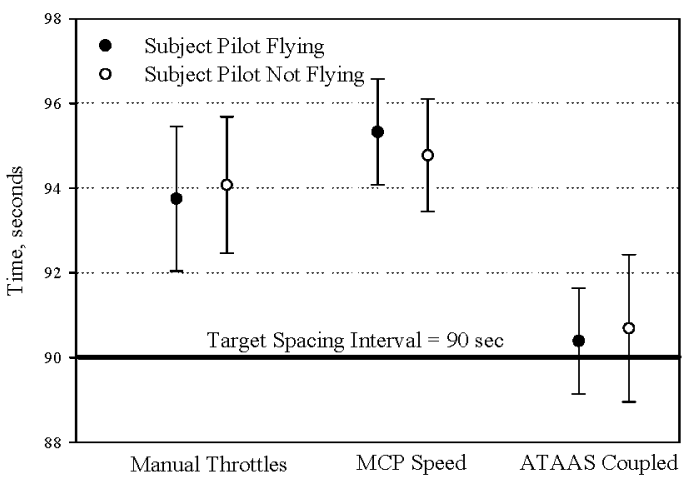

Figure 9. Piloted simulation runwoy threshold spacing interval results.

minimal changes in scan pattern and no significant increase in heads-down time as a result of using the ATAAS tool.

\section{Planned flight research}

A flight activity is planned for the near future in which three aircraft of differing performance characteristics will fly the approach spacing concept. The objective of these flights is to validate of the results of the simulator study in an operational environment. In addition to the RNAV routes flown in the study, other scenarios planned for the flight activity will demonstrate the flexibility of the spacing tool. These scenarios are based on a lead aircraft receiving vectors and subsequent aircraft following time history trails of the immediately preceding aircraft.

\section{Merging and Maneuvering Operations}

Merging operations will be investigated through algorithm and display modifications to support the capability to meet RTAs. A major concern for merging operations is that aircraft on the same or closely spaced routes may use different strategies to meet times at the merge point, which could result in conflicts

Maneuvering operations in the terminal area require additional work in several areas, including flight crew information requirements, aircraft equipment requirements, and procedures. Future work in developing a maneuvering capability is anticipated to utilize results of research and development that is currently being conducted for the en-route domain. The AOP will be extended to provide trajectories that enable maneuvering within prescribed corridors.

\section{$\underline{\text { Feasibility }}$}

Research and development to date provides strong evidence that free flight operations in capacityconstrained terminal environments are feasible and 
provide benefits. In-trail approach spacing operations can be performed under real-world conditions with minimal impacts to crew workload.

Research will continue to quantify the benefits achievable with terminal area free flight operations. Some of the benefits that have already been identified in both safety and efficiency can be manifested in a number of ways. For example, flight deck alerting of predicted separation violations (generated by airborne algorithms) can augment those provided by the ATSP. Safer operations can also result from less frequency congestion, and through a mitigation of errors based on a redistribution of workload between pilots and controllers.

\section{$\underline{\text { Conclusions }}$}

Although research is not yet complete, findings indicate that free flight is feasible and may significantly enhance system capacity and safety. The research has improved the understanding of free flight feasibility issues well beyond that used to develop the early mixed assessments. Plausible technical solutions to all identified free flight issues either have been developed or envisioned. Research completed to date has led to the following conclusions:

- Airborne autonomous operations can be reliably performed without controller intervention in the en route domain.

- Pilot workload in nominal en-route situations is not a feasibility issue, even for very high traffic densities. Therefore, free flight operations in the en route domain scale well with traffic density.

- Implicit coordination of conflict resolution maneuvers rather than negotiation between aircraft is sufficient for airborne separation assurance

- Tactical traffic conflict resolution actions do not cause a significant loss of conformance with RTA time constraints in en route airspace, even for descents. Therefore, a simple state-based conflict detection and resolution system may have a high benefit/cost ratio.

- Given the appropriate flight deck decision support automation, pilots can strategically resolve conflicts in highly constrained environments, including those containing flow, airspace, and performance constraints.

- The exchange of intent information between aircraft is not a fundamental requirement for feasibility, but the combined use of state and intent provides valuable operational benefits.

- Airborne separation assurance in terminal arrival environments is feasible, but highly constrained terminal environments with merging arrival streams will probably involve limited delegation of responsibility by the ATSP and require more sophisticated airborne tools than currently exist, especially for maximizing airport throughput.

- Airborne approach spacing for all aircraft on a common approach path is feasible, and it provides significant capacity benefits due to increased flying precision. The impact of such operations on pilot workload is minor.

Research to date also suggests that a feasible, acceptable, and viable free flight concept needs to accommodate a wide range of airborne capability within many operating environments. It should be possible to fill these needs through the combined use of tactical and strategic airborne decision support automation. There is a role for tactical systems for airspace users who do not have the economic incentive for strategic automation. Tactical systems also may be most appropriate for providing a certifiably safe conflict resolution function, and they facilitate a transition to future operations by providing a limited capability at low-cost. Strategic systems may be most appropriate for airspace users that desire to optimize their trajectories in airspace environments dominated by severe flow management and airspace constraints, or in environments prone to high crew workload. These systems may also be crucial for environments that require air/ground integration as part of a traffic management solution that integrates autonomous and managed aircraft operations. Research conducted so far also suggests that the most preferred airborne system will be one that combines both tactical and strategic functions.

Much research and development remains to understand limits of feasibility and develop enabling technologies. Ongoing activities are focusing on

- Analysis of free flight in extremely constrained situations and airspace domains.

- Technology and procedures for distributed air/ground operations in integrated mixedequipage airspace environments.

- Airborne merging and maneuvering integrated with airborne spacing in the terminal environment.

- Transition of responsibility between air and ground participants under nominal and failuremode scenarios.

- Continued development and refinement of airborne decision support technology and the establishment of requirements and standards for 
the enabling CNS infrastructure.

- Detailed analysis of safety and economic viability of DAG-TM concepts.

The future approach will include the simulation of DAG-TM air and ground components through interconnected traffic simulation laboratories at Langley, Ames, and the NLR. The Ames laboratory will simulate the ground-based components of the concepts, including the air traffic service provider and aeronautical operational control, while the Langley and NLR laboratories will simulate the airborne components. Monte Carlo analyses are planned to further investigate safety and the impacts of reducing current separation standards. The analyses will utilize multiple instantiations of the airborne technology developed for human-in-the-loop investigations and human operator models rather than simplified systemlevel representations of these functions. Full-mission flight simulations will be used to develop multi-crew procedures and evaluate crew workload. Airborne validation of the developed concepts and technologies is also needed as part of a comprehensive proof of feasibility.

Because of its inherent advantages in increasing system capacity and safety, free flight should be considered an essential part of a comprehensive air traffic management modernization activity. While free flight cannot alone resolve the complex issues faced by those modernizing the global airspace, it can be pivotal in providing a scalable and redundant solution for all phases of flight between pushback and gate arrival.

\section{$\underline{\text { References }}$}

1. Final Report of the RTCA Task Force 3: Free Flight Implementation. RTCA, Inc., Washington DC, October 1995.

2. NASA Advanced Air Transportation Technologies Project: Concept Definition for Distributed Air/Ground Traffic Management (DAG-TM), Version 1.0, September 1999.

3. Wickens, C. D.; Mavor, A. S.; and McGee, J. P., ed.: Flight to the Future: Human Factors in Air Traffic Control. National Academy Press, Washington, D.C., 1997.

4. Wickens, C.D., Mavor, A.S., Parasuraman, R., and McGee, J.P., ed.: The Future of Air Traffic Control: Human Operators and Automation. National Academy Press: Washington, DC, 1998.

5. Hoekstra, J. M.; van Gent, R. N. H. W.; and Ruigrok, R. C. J.: Conceptual Design of Free Flight with Airborne Separation Assurance. AIAA-98-4239, August 1998.

6. Eby, M. S., and Kelly III, W. E.: Free Flight Separation Assurance Using Distributed Algorithms. Proceedings
CD: Entry \# 4.302, ISBN \# 0-7803-5427-3, IEEE Aerospace Conference, March 1999.

7. Ballin, M. G., Sharma, V., Vivona, R., Johnson, E. J., and Ramiscal, E.: A Flight Deck Decision Support Tool for Autonomous Airborne Operations. AIAA2002-4554, August 2002.

8. Mondoloni, S., Wing, D. J., and Palmer, M. T.: Development of a Prototype Airborne Conflict Detection and Resolution Simulation Capability. AIAA-2002-4446, August 2002.

9. Eby, M.S.: A Self-Organizational Approach for Resolving Air Traffic Conflicts. The Lincoln Laboratory Journal, MIT, Vol. 7, No. 2, 1994, pages 239-254.

10. Hoekstra, J. M.: Designing for Safety: the Free Flight Air Traffic Management. ISBN 90-806343-2-8, National Aerospace Laboratory NLR, Netherlands, November 2001.

11. Zijlstra, F. R. H., and van Doom, L.: The Construction of a Scale to Measure Subjective Effort. Technical Report, Delft University of Technology, Department of Philosophy and Social Sciences, The Netherlands, 1985.

12. Boeing Commercial Aircraft Group: Statistical Summary of Commercial Jet Aircraft Accidents, Worldwide Operations 1959-1994. The Boeing Commercial Airplane Group, 1994.

13. Wing, D. J.; Adams, R. J.; Duley, J. A.; Legan, B. M.; Barmore, B. E., and Moses, D.: Airborne Use of Traffic Intent Information in a Distributed Air-Ground Traffic Management Concept: Experiment Design and Preliminary Results. NASA TM-2001-211254, 2001.

14. Wing, D J.; Barmore, B. E.; and Krishnamurthy, K.: Use of Traffic Intent Information by Autonomous Aircraft in Constrained Operations. AIAA-2002-4555, August 2002.

15. Williams, D. H.: Time-Based Self-Spacing Techniques Using Cockpit Display of Traffic Information During Approach to Landing in a Terminal Area Vectoring Environment. NASA TM84601, April 1983

16. Williams, D. H., and Wells, D. C.: Jet Transport Flight Operations Using Cockpit Display of Traffic Information During Instrument Meteorological Conditions, Simulation Evaluation. NASA TP-2567, May 1986.

17. Abbott, T. S.: Speed Control Law For Precision Terminal Area In-Trail Self-Spacing. NASA TM2002-211742, July 2002.

18. Hart, S. G., and Staveland, L. E.: Development of a Multi-Dimensional Workload Rating Scale: Results Of Empirical and Theoretical Research. In P. A. Hancock \& N. Meshkati, ed.: Human Mental Workload, 139-183. Amsterdam, The Netherlands, Elsevier, 1988. 